\title{
Seropositivity of Scrub Typhus Inpatients Attending a Tertiary Care Hospital in Western Odisha
}

\author{
Shuvankar Mukherjee ${ }^{1}$, Anshuman Dash², Shreekant Tiwari ${ }^{3}$ \\ 1Department of Microbiology, Hitech Medical College and Hospital, Rourkela, Odisha, India. ${ }^{2}$ Department of \\ Microbiology, Hitech Medical College and Hospital, Rourkela, Odisha, India. ${ }^{3}$ Department of Microbiology, Hitech \\ Medical College and Hospital, Rourkela, Odisha, India.
}

\section{ABSTRACT}

\section{BACKGROUND}

Scrub typhus is a vector borne Rickettsial disease caused by Orientia tsutsugamushi and has been reported from tsutsugamushi triangle which includes India. It is a reemerging pathogen in India including the state of Odisha. This study was conducted to determine seropositivity of scrub typhus in patients attending a tertiary care hospital of Western Odisha.

\section{METHODS}

Fifty febrile patients who were negative for Malaria, Dengue and Leptospira attending a tertiary care hospital of Western Odisha were enrolled in the study. $3 \mathrm{ml}$ of blood was collected, and serum was separated. IgM SD Bioline Tsutsugamushi lateral flow immunochromatography test was performed as per manufacturer instruction. Positive samples were confirmed by PCR method (Geno-Sen's ST Real Time PCR).

\section{RESULTS}

$20 \%$ of patients were positive for IgM SD Bioline Tsutsugamushi card test. $60 \%$ of positive patients were male. $20 \%$ of positive patients were having pathognomonic eschar. $60 \%$ of infected patients were in the age group of $10-30$.

\section{CONCLUSIONS}

In this study, $20 \%$ of febrile patients were positive for Scrub typhus infection. So, it should be included in the differential diagnosis of a febrile patient in Western Odisha.

\section{KEY WORDS}

Scrub Typhus, Seropositivity, Western Odisha, Card Test.
Corresponding Author:

Shuvankar Mukherjee,

Staff Quarters, Room No. 28,

Hitech Medical College and Hospital,

Rourkela769004, Odisha, India

E-mail: shuvankar.mukherjee@gmail.com

DOI: $10.14260 /$ jemds/2020/42

Financial or Other Competing Interests: None.

How to Cite This Article:

Mukherjee $S$, Dash A, Tiwari $S$. Seropositivity of scrub typhus inpatients attending a tertiary care hospital in Western Odisha. J. Evolution Med. Dent. Sci. 2020;9(04):178-181, 10.14260/jemds/2020/42 DOI:

Submission 11-11-2019,

Peer Review 07-01-2020,

Acceptance 14-01-2020,

Published 27-01-2020. 


\section{BACKGROUND}

Scrub typhus also known as tsutsugamushi disease caused by Orientia tsutsugamushi (Tsutsuga means something small and dangerous and mushi means insect.) ${ }^{1}$ is widely prevalent in Asia pacific area, so called tsutsugamushi triangle, a geographical region comprising of South East Asia and South west pacific. ${ }^{2}$ Annually, one million Scrub typhus cases have been reported in these region, and one billion individual are at risk of contracting the illness. ${ }^{3}$ It causes acute undifferentiated fever with common symptoms such as fever, rash, nausea, vomiting, thrombocytopenia, ${ }^{4}$ so it can be confused easily with other diseases like Malaria, Dengue, Chikungunya, Enteric fever and Leptospirosis. Complication occur in $4 \%$ of patients like pneumonitis, meningoencephalitis, jaundice, renal failure and myocarditis. Patient usually die due to multi organ failure.5,6 Previous study showed that scrub typhus was apparent in the endemic region for short time. Only sporadic data are available regarding scrub typhus in different areas, resulting in a gap of knowledge about the disease. The tsutsugamushi triangle. ${ }^{7}$ is a area covering more than 8 million $\mathrm{Km}^{2}$, from the Russian Far East to Australia, and from Pakistan to the Japan. ${ }^{7}$ As this area is densely populated about one billion people are at risk of infection. Even the non-endemic region becomes vulnerable due to export of infection by air travel and globalisation. Epidemiological study of scrub typhus is difficult due to antigenic and genetic diversity of $O$. tsutsugamushi strains. It was popular as typhus like fever in India before 1917. Many military personnel of world war IIalong Burma border and 1965 Indo Pak war suffered fever due to this disease. ${ }^{7}$ In 1990 scrub typhus came back with full virulence at the Pakistan border of India. ${ }^{7}$ Insecticides and empiric antibiotic therapy decreased the disease prevalence thereafter in civilian life. However, scrub typhus is not a well diagnosed disease in India. Epidemiological data say that the disease is prevalent all over India. Maharashtra, Tamil Nadu, Karnataka, Kerala, Himachal Pradesh, Jammu and Kashmir, Uttaranchal, Rajasthan, Odisha, West Bengal, Bihar, Meghalaya, and Nagaland 7 have reported the incidence of scrub typhus. Post monsoon august to October is the season of scrub typhus due to humid environment. Attack rate of Orientia is more in uneducated and rural population of India.

Scrub typhus is not always benign, sometimes it causes lot of complication. Multiorgan failure involving liver kidney, lung, heart, brain, vascular system leading to DIC and death has been reported.7 Primary target site of Orientia is lung so ARDS, Interstitial pneumonia are common. Meningoencephalitis leads to agitation, disorientation or even coma. Focal neurological involvement also can occur. CSF of patient of meningitis due to scrub typhus resembles that of TB or viral meningitis. Inner ear involvement leads to hearing impairment. Marked hyperaemia, haemorrhage on conjunctiva, GI mucosal haemorrhage and coagulation disorder including DIC can also be seen. In recent years scrub typhus has rapidly re-emerged to become important cause of PUO in different parts of India. 23 states of India have reported the presence of Scrub typhus and Odisha is one of them. $8,9,10,11$ Case fatality varies in different region ranging from 0 to $100 \%$. However median case fatality rate is $1.4 \%$ and $6 \%$ in treated and untreated patient respectively. ${ }^{7}$
Therefore, development of effective measure to prevent, control and treat scrub typhus is an urgent health need of Indian community and to do this it is very much important to know local seropositivity rate of scrub typhus in different region of India. Keeping it in mind we have conducted a research to know seropositivity rate of Scrub typhus in patient with undifferentiated fever attending a tertiary care hospital of Western Odisha.

\section{METHODS}

It was a cross sectional study. Fifty (50) febrile patients attending in tertiary care hospital in Western Odisha from May 2019 to October 2019, who were negative for Malaria (by thin smear and thick smear examination) and Dengue (Dengue IgM ELISA) were enrolled in the study. Under aseptic precaution $3 \mathrm{ml}$ of blood was collected and sera was separated, and sera was subjected to SD Bioline tsutsugamushi lateral flow immunochromatography test. 50 ul of serum put into sample well and one drop of buffer was added. Result was read after 15 minutes. Control band and test band if both were present the test was declared positive. Positive samples were confirmed by Positive samples were confirmed by PCR method (Geno-Sen's ST Real Time PCR).

\section{Statistical Analysis}

SPSS Software Ver. 16 was used for analysis of data.

\section{RESULTS}

A total of 50 febrile patients who were negative for Malaria, Dengue and Leptospira were enrolled. Their age ranged from 10 to 65 years. $60 \%(30 / 50)$ were male. $20 \%(10 / 50)$ were positive for IgM antibodies against 0 . tsutsugamushi. $60 \%$ (6/10) of positive patients were Male. 20\% (2/10) of positive patient had eschar. Higher seropositivity of Scrub typhus; $60 \%(6 / 10)$ was seen in age group of $10-30$ followed by $31-50$ age group (30\%) and 51-65 age group (10\%).10 Samples positive for card test were confirmed by ELISA IgM assay. (In Bios International Inc., USA).

\begin{tabular}{|c|c|c|c|}
\hline Gender & No. of Patients & Scrub Typhus +ve & Scrub Typhus -ve \\
\hline Male & 30 & 6 & 24 \\
\hline Female & 20 & 4 & 16 \\
\hline Total & $\mathbf{5 0}$ & 10 & $\mathbf{4 0}$ \\
\hline \multicolumn{2}{|c|}{ Table 1. Scrub Typhus Positivity among Male and Female } \\
\hline
\end{tabular}

\begin{tabular}{|c|c|c|}
\hline Age Group & No. of Patients & Scrub Typhus Positive \\
\hline $10-30$ & 22 & 6 \\
\hline $31-50$ & 21 & 3 \\
\hline $51-60$ & 7 & 1 \\
\hline \multicolumn{2}{|c|}{ Table 2 Scrub Typhus Positivity in Different Age Groups } \\
\hline
\end{tabular}

\section{DISCUSSION}

Chiggers or Leptotrombidium mites are the vector and primary reservoir of Orientia tsutsugamushi.$^{12}$ Mites remains infected throughout their life cycle. ${ }^{13}$ Chiggers or larva usually feed once on thin, tender, wrinkled skin for 2 to 3 
days. Chiggers release some enzyme like material through saliva and dissolve host tissue around hair follicle but does not pierce skin. Orientia is found in salivary gland of infected mite. Both transovarian and transstadial transmission maintain Orientia in mites. ${ }^{12,13}$ Wild rodents can also be a source of infection to mites. As Orientia is an intracellular bacterium it is very much difficult to study mechanism of pathogenesis. Reinfection can occur due to short lived immunity and antigenic heterogeneity. However, data regarding epidemiology and pathogenesis of scrub typhus is scanty in literature compared to another disease. .,14 $^{2}$

According to World health organisation "Scrub typhus is probably one of the most underdiagnosed and under reported febrile illness requiring hospitalization. In our study seropositivity of Scrub typhus was $20 \%$ which was higher than Rao et al $^{15}$ (2019), Raghunath et al ${ }^{16}$ (2018) Lalrinkima et $\mathrm{al}^{1}$ (2017), Oberoi et $\mathrm{al}^{17}$ (2019) Pote et al ${ }^{18}$ (2018) but lower than Madhusmita et $\mathrm{al}^{19}$ (2019), Sahu et al ${ }^{20}$ (2015) Saramma et $\mathrm{al}^{21}$ (2019), Rizvi et $\mathrm{al}^{22}$ (2019), Khan et $\mathrm{al}^{23}$ and Jhakaria et al. ${ }^{24}$ In our study Male affected more than female (60\% vs $40 \%$ ) Similar distribution was seen in Madhusmita et $\mathrm{al}^{19}(61.7 \%)$, Palas Das et $\mathrm{al}^{25}(58 \%)$, Raghunath et $\mathrm{al}^{25}$ (61\%) Lalrinkima et al $^{1}(56.6 \%)$ but female more than male distribution was seen in Saramma et al ${ }^{21}(65 \%$ female $)$, Rajendra et $\mathrm{al}^{26}$ (64\% female)). It may be due to fact that in this part of Odisha male are more involved in agricultural works than female. About age distribution our study showed that younger generation are affected more than older generation $(60 \%$ in age group of $10-30,30 \%$ in age group of 31-50 and $10 \%$ in age group of 51-65) similar finding was reported by Saramma et al, ${ }^{21}$ Raghunath et al, ${ }^{16}$ Lalrinkima et al, ${ }^{1}$ Rajendra et al ${ }^{26}$ and Rizvi et al. ${ }^{22}$

Eschar is papuloulcerative ${ }^{13}$ lesion with black crust which is the classic clinical feature formed at the site of mite feeding. It develops before fever onset and another symptom. It is found in 1 to $97 \%$ of patients depending upon geographical area. It is more prominent in white skinned people compared to dark skinned Indians ${ }^{7}$. Most common sites of eschar in male are abdomen specially $30 \mathrm{~cm}$ below the umbilicus followed by lower extremity and anterior chest. In female anterior chest and head neck are most commonly involved sites and in children axilla is the commonest site. ${ }^{13}$

In our study most pathognomonic sign eschar was found in $20 \%$ of positive patient which is higher than Madhusmita et $\mathrm{al}^{19}(17.9 \%)$, Patnayak et $\mathrm{al}^{27}(12 \%)$, Saramma et $\mathrm{al}^{21}$ (8.7\%), Navneet et $\mathrm{al}^{28}$ (145), Christpal et $\mathrm{al}^{29}$ (4.5\%), Rajendra et $\mathrm{al}^{26}(12 \%)$, Rizvi et $\mathrm{al}^{22}(0 \%)$ but lower than Patnayak et $\mathrm{al}^{27}(48 \%)$, Das et $\mathrm{al}^{25}(27.74 \%)$ and Sahu et $\mathrm{al}^{20}$ (32\%).

In India, the first case of scrub typhus was reported from Assam and West Bengal in the year 1945 and re-emergence of this was reported in 2010 after a gap of 65 years. Since then there are paucity of data about seropositivity of scrub typhus in different part of India. ${ }^{30}$ In Odisha it varies from $3.5 \%$ to $48.7 \%$. An eschar is not found in all cases of Scrub typhus, local seropositivity rate of Scrub typhus is very important information for clinician to rule out scrub typhus in undifferentiated fever and to formulate preventive strategy.

\section{CONCLUSIONS}

In this study $20 \%$ of febrile patients were positive for scrub typhus infection. $20 \%$ of infected patients showed pathognomonic eschar. Among scrub typhus positive patients $60 \%$ were male and $40 \%$ were female. It should be included in the differential diagnosis of a febrile patient in Western Odisha. Preventive measures like protective clothing and application of insect repellents along with public awareness about the disease transmission dynamics are important for reducing the disease prevalence.

\section{Limitations}

This study was limited by small sample size and the fact that it relied upon lateral flow card test not by ELISA IgM assay. However, a correlation of $97 \%$ between IgM ELISA and SD Bioline Tsutsugamushi rapid test was reported In India. ${ }^{31} \mathrm{~A}$ community-based study with a large sample size using ELISA IgM will give a better understanding of the prevalence and incidence of scrub typhus in Western Odisha.

\section{REFERENCES}

[1] Lalrinkima $H$, Lalremruata $R$, Lalchhandama $C$, et al. Scrub typhus in Mizoram, India. Journal of Vector Borne Diseases 2017;54(4):369-71.

[2] Chunchanur SK. Scrub typhus in India - an impending threat! Ann Clin Immunol Microbiol 2018;1(1):1003.

[3] Bonell A, Lubell Y, Newton PN, et al. Estimating the burden of scrub typhus: a systematic review. PLoS Negl Trop Dis 2017;11(9):e0005838.

[4] Hun L, Troyo A. An update on the detection and treatment of Rickettsia felis. Res Rep Trop Med 2012;3:47-55

[5] Kawamura A. Tsutsugamushi disease: an overview. In: Kawamura A Jr, Tanaka H, Tamura A, eds. Tsutsugamushi Disease. Tokyo, Japan: University of Tokyo Press 1995: p. 1-34.

[6] Sonthayanon P, Chierakul W, Wuthiekanun V, et al. Rapid diagnosis of scrub typhus in rural Thailand using polymerase chain reaction. Am J Trop Med Hyg 2006;75(6):1099-102.

[7] Xu G, Walker DH, Jupiter D, et al. A review of the global epidemiology of scrub typhus. PLoS Negl Trop Dis 2017;11(11):e0006062.

[8] Trowbridge P, Divya P, Prasanna PS, et al. Prevalence and risk factors for scrub typhus in South India. Trop Med Int Health 2017;22(5):576-82.

[9] Laskar AR, Suri S, Acharya AS. Scrub typhus: Reemerging public health problem in India. J Commun Dis 2015;47(3):19-25.

[10] Jeong MA, Youn SK, Kim YK, et al. Trends in the incidence of scrub typhus: the fastest growing vector-borne disease in Korea. Osong Public Health Res Perspect 2013;4(3):166-9.

[11] Rahi M, Gupte MD, Bhargava A, et al. DHR-ICMR guidelines for diagnosis \& management of Rickettsial diseases in India. Indian J Med Res 2015;141(4):417-22. 
[12] Phasomkusolsil S, Tanskul P, Ratanatham S, et al. Influence of Orientia tsutsugamushi infection on the developmental biology of Leptotrombidium imphalum and Leptotrombidium chiangraiensis (Acari: Trombiculidae). J Med Entomol 2012;49(6):1270-5.

[13] Jeong YJ, Kim S, Wook YD, et al. Scrub typhus: clinical, pathologic and imaging findings. Radiographics 2007;27(1):161-72.

[14] Peter JV, Sudarsan TI, Prakash JA, et al. Severe scrub typhus infection: clinical features, diagnostic challenges and management. World J Crit Care Med 2015;4(3):24450.

[15] Rao P, Van Eijk A, Choubey S, et al. Dengue, Chikungunya and scrub typhus are important etiologies of nonmalarial febrile illness in Rourkela, Odisha, India. BMC Infectious Diseases 2019;19(1):572.

[16] Raghunath P, Paul PJ, Oommen S, et al. Detection of scrub typhus at a tertiary health centre in Central Kerala. J Acad Clin Microbiol 2018;20(1):28-32.

[17] Oberoi A, Varghese SR. Scrub typhus-an emerging entity: a study from a tertiary care hospital in North India. Indian J Public Health 2014;58(4):281-3.

[18] Pote K, Narang R, Deshmukh P. Diagnostic performance of serological tests to detect antibodies against acute scrub typhus infection in central India. Indian J Med Microbiol 2018;36(1):108-12.

[19] Bal M, Mohanta MP, Sahu S, et al. Profile of pediatric scrub typhus in Odisha, India. Indian Pediatrics 2019;56(4):304-6.

[20] Sahu S, Misra SR, Padhan P, et al. Scrub typhus in a tertiary care hospital in the eastern part of Odisha. Apollo Medicine 2015;12(1):2-6.

[21] Jacob SM, Sekkizhar G, Kanagasabai S, et al. Seroprevalence and clinical manifestations of scrub typhus infection in Chennai city: a cross-sectional study. Int J Health Allied Sci 2018;7(3):201-3.
[22] Rizvi M, Sultan A, Chowdhry M, et al. Prevalence of scrub typhus in pyrexia of unknown origin and assessment of interleukin-8, tumor necrosis factor-alpha and interferon-gamma levels in scrub typhus-positive patients. Indian J Pathol Microbiol 2018;61(1):76-80.

[23] Khan SA, Khamo V, Uriah HJ, et al. Scrub typhus in hilly regions of northeast India. Indian $\mathrm{J}$ Med Res 2016;144(1):138-40.

[24] Jakharia A, Borkakoty B, Biswas D, et al. Seroprevalence of scrub typhus infection in Arunachal Pradesh, India. Vector Borne Zoonotic Dis 2016;16(10):659-63.

[25] Das P, Singh D, Das M, et al. Epidemiological and clinical features of scrub typhus in Odisha, Eastern India. Med J DY Patil Vidyapeeth 2019;12(5):419-23.

[26] Takhar RP, Bunkar ML, Arya S, et al. Scrub typhus: a prospective, observational study during an outbreak in Rajasthan, India. The National Medical Journal of India 2017;30(2):69-72.

[27] Patnaik S, Swain N, Sahoo B, et al. Emergence of scrub typhus in Odisha - a hospital based study. Ann Trop Med Public Health 2017;10(3):636-40.

[28] Sharma N, Biswal M, Kumar A, et al. Scrub typhus in a tertiary care hospital in North India. The American Journal of Tropical Medicine and Hygiene 2016;95(2):447-51.

[29] Chrispal A, Boorugu H, Gopinath KG, et al. Scrub typhus: an unrecognized threat in South India - clinical profile and predictors of mortality. Trop Doct 2010;40(3):12933.

[30] Davis GE, Austrian RC, Bell EJ. Observations on tsutsugamushi disease (scrub typhus) in Assam and Burma: The recovery ofstrains of Rickettsia Orientialis. Am J Trop Med Hyg 1947;46(2):268-86.

[31] Ramyasree A, Kalawat U, Rani ND, et al. Seroprevalence of scrub typhus at a tertiary care hospital in Andhra Pradesh. Indian J Med Microbiol 2015;33(1):68-72. 